\title{
ON SPECTRAL PROPERTIES OF TRANSLATIONALLY INVARIANT MAGNETIC SCHRÖDINGER OPERATORS
}

\author{
D. YAFAEV
}

\begin{abstract}
We consider a class of translationally invariant magnetic fields such that the corresponding potential has a constant direction. Our goal is to study basic spectral properties of the Schrödinger operator $\mathbf{H}$ with such a potential. In particular, we show that the spectrum of $\mathbf{H}$ is absolutely continuous and we find its location. Then we study the long-time behaviour of solutions $\exp (-i \mathbf{H} t) f$ of the time dependent Schrödinger equation. It turnes out that a quantum particle remains localized in the plane orthogonal to the direction of the potential. Its propagation in this direction is determined by group velocities. It is to a some extent similar to a evolution of a one-dimensional free particle but "exits" to $+\infty$ and $-\infty$ might be essentially different.
\end{abstract}

\section{INTRODUCTION}

1.1. Traslationally invariant magnetic fields $B(x)=\left(b_{1}(x), b_{2}(x), b_{3}(x)\right), x=\left(x_{1}, x_{2}, x_{3}\right)$, $\operatorname{div} B(x)=0$, give important examples where a non-trivial information can be obtained about spectral properties of the corresponding Schrödinger operators $\mathbf{H}$. We suppose for definiteness that $B(x)$ does not depend on the $x_{3}$-variable so that $\mathbf{H}$ commute with translations along the $x_{3}$-axis. There are two essentially different (and in some sense extreme) classes of traslationally invariant magnetic fields.

The first class consists of fields $B(x)=\left(0,0, b_{3}\left(x_{1}, x_{2}\right)\right)$ of constant direction. For such fields, the momentum $p$ of a classical particle in the $x_{3}$-direction is conserved, and in the Schrödinger equation the variable $x_{3}$ can be separated. Thus, we arrive to a two-dimensional problem in the $\left(x_{1}, x_{2}\right)$-plane. Furthermore, if $b_{3}$ is a function of $r=\left(x_{1}^{2}+x_{2}^{2}\right)^{1 / 2}$ only, then we get a set of problems on the half-line $r>0$ labelled by the magnetic quantum number $m$. The most important example of this type is a constant magnetic field $b_{3}(r)=$ const (see [10]). Some class of functions $b_{3}(r)$ decaying as $r \rightarrow \infty$ was discussed in [12] (see also [2]) where new interesting effects were found. Another famous case is $b_{3}\left(x_{1}, x_{2}\right)=\delta\left(x_{1}, x_{2}\right)(\delta(\cdot)$ is the Dirac delta-function) studied in 11. Scattering by an arbitrary short-range (decaying faster than $|x|^{-2-\varepsilon}, \varepsilon>0$, as $|x| \rightarrow \infty)$ magnetic field $b_{3}\left(x_{1}, x_{2}\right)$ turns out to be rather similar to this particular case (see [16]).

The second class consists of fields $B(x)=\left(b_{1}\left(x_{1}, x_{2}\right), b_{2}\left(x_{1}, x_{2}\right), 0\right)$ orthogonal to the $x_{3}$-axis. In this case the corresponding magnetic potential $A(x)$, defined (up to gauge

2000 Mathematics Subject Classification. 47A40, 81U05.

Key words and phrases. magnetic fields, translation invariance, spectral theory, dispersion curves, group velocities, long-time evolution. 
transformations) by the equation curl $A(x)=B(x)$, can be chosen as

$$
A(x)=\left(0,0,-a\left(x_{1}, x_{2}\right)\right)
$$

so that it has the constant direction. In contrast to fields of the first class, now the variable $x_{3}$ cannot be separated in the Schrödinger equation. Nevertheless due to the invariance with respect to translations along the $x_{3}$-axis the operator (we always suppose that the charge of a particle is equal to 1 )

$$
\mathbf{H}=(i \nabla+A(x))^{2}
$$

can be realized, after the Fourier transform in the variable $x_{3}$, in the space $L^{2}\left(\mathbb{R} ; L^{2}\left(\mathbb{R}^{2}\right)\right)$ as the operator of multiplication by the operator-valued function

$$
H(p)=-\Delta+\left(a\left(x_{1}, x_{2}\right)+p\right)^{2}: L^{2}\left(\mathbb{R}^{2}\right) \rightarrow L^{2}\left(\mathbb{R}^{2}\right), \quad p \in \mathbb{R}
$$

Moreover, if $a\left(x_{1}, x_{2}\right)=a(r)$, then the subspaces with fixed magnetic quantum number $m \in \mathbb{Z}$ are invariant subspaces of $H(p)$ so that the operator $H(p)$ reduces to the orthogonal sum over $m \in \mathbb{Z}$ of the operators

$$
H_{m}(p)=-\frac{1}{r} \frac{d}{d r} r \frac{d}{d r}+\frac{m^{2}}{r^{2}}+(a(r)+p)^{2}
$$

acting in the space $\mathcal{H}=L^{2}\left(\mathbb{R}_{+} ; r d r\right)$. In this case the field is given by the equation

$$
B(x)=b(r)(-\sin \theta, \cos \theta, 0)
$$

where $b(r)=a^{\prime}(r)$ and $\theta$ is the polar angle. Thus, vectors $B(x)$ are tangent to circles centered at the origin. An important example of such type is a field created by a current along an infinite straight wire (coinciding with the $x_{3}$-axis). In this case $b(r)=b_{0} r^{-1}$ so that $a(r)=b_{0} \ln r$. The Schrödinger operator with such magnetic potential was studied in [15].

1.2. In this article we consider magnetic fields (1.5) with a sufficiently arbitrary function $b(r)$. Our goal is to study basic spectral properties of the corresponding Schrödinger operator $\mathbf{H}$ such as the absolute continuity, location and multiplicity of the spectrum, as well as the long-time behaviour of the unitary group $\exp (-i \mathbf{H} t)$. We emphasize that for magnetic fields considered here, the problem is genuinely three-dimensional, and actually the motion of a particle in the $x_{3}$-direction is of a particular interest.

Using the cylindrical invariance of field (1.5), we can start either from translational or from rotational (around the $x_{3}$-axis) symmetries. The rotational invariance implies that the operator $\mathbf{H}$ is the orthogonal sum of its restrictions $\mathbf{H}_{m}$ on the subspaces of functions with magnetic quantum number $m \in \mathbb{Z}=\{0, \pm 1, \pm 2, \ldots\}$. It can be identified with the operator (we keep the same notation for this operator)

$$
\mathbf{H}_{m}=-\frac{1}{r} \frac{d}{d r} r \frac{d}{d r}+\frac{m^{2}}{r^{2}}+\left(i \frac{d}{d x_{3}}+a(r)\right)^{2}
$$

acting in the space $\mathfrak{H}=L^{2}\left(\mathbb{R}_{+} \times \mathbb{R} ; r d r d x_{3}\right)$. In view of the translation invariance, every operator $\mathbf{H}_{m}$ can be realized (again after the Fourier transform in the variable $x_{3}$ ) in the space $L^{2}\left(\mathbb{R} ; L^{2}\left(\mathbb{R}_{+} ; r d r\right)\right)$ as the operator of multiplication by the operator-valued function $H_{m}(p)$ defined by (1.4). 
Suppose now that $b(r)$ does not tend to zero too fast so that $a(r) \rightarrow \infty$ as $r \rightarrow \infty$. Then the spectrum of each operator $H_{m}(p)$ is discrete. Let $\lambda_{n, m}(p), n \in \mathbb{N}=\{1,2, \ldots\}$, be the increasing sequence of its eigenvalues (they are simple and positive), and let $\psi_{n, m}(r, p)$ be the corresponding sequence of its eigenfunctions. The functions $\lambda_{n, m}(p)$ are known as dispersion curves of the problem. They determine the spectral properties of the operator $\mathbf{H}_{m}$.

Note that if $a(r)$ is replaced by $-a(r)$, then $\lambda_{n, m}(p)$ is replaced by $-\lambda_{n, m}(p)$, so that the case $a(r) \rightarrow-\infty$ as $r \rightarrow \infty$ is automatically included in our considerations. Recall that, for a magnetic field $B(x)$, the magnetic potential $A(x)$ such that curl $A(x)=B(x)$ is defined up to a gauge term grad $\varphi(x)$. In particular for magnetic fields (1.5) in the class of potentials $A(x)=(0,0,-a(r))$ one can always add to $a(r)$ an arbitrary constant $c$. This leads to the transformations $\lambda_{n, m}(p) \mapsto \lambda_{n, m}(p-c)$ and $\psi_{n, m}(r, p) \mapsto \psi_{n, m}(r, p-c)$.

1.3. The precise definitions of the operators $\mathbf{H}_{m}$ and $\mathbf{H}$ and their decompositions into the direct integrals over the operators $H_{m}(p)$ and $H(p)$ are given in Section 2. To put it differently, we construct a complete set of eigenfunctions of the operator $\mathbf{H}$. They are parametrized by the magnetic quantum number $m$, the momentum $p$ in the direction of the $x_{3}$-axis and the number $n$ of an eigenvalue $\lambda_{m, n}(p)$ of the operator $H_{m}(p)$. Thus, if we set

$$
\boldsymbol{\psi}_{n, m, p}\left(r, \theta, x_{3}\right)=e^{i p x_{3}} e^{i m \theta} \psi_{n, m}(r, p)
$$

then

$$
\mathbf{H} \boldsymbol{\psi}_{n, m, p}=\lambda_{n, m}(p) \boldsymbol{\psi}_{n, m, p} .
$$

In Section 3, we show that for all $n \in \mathbb{N}$ and $m \in \mathbb{Z}$ :

- Under very general assumptions $\lambda_{n, m}(p) \rightarrow \infty$ as $p \rightarrow \infty$ (Proposition 3.3).

- If $b(r) \rightarrow 0$ as $r \rightarrow \infty$, then $\lambda_{n, m}(p) \rightarrow 0$ as $p \rightarrow-\infty$ (Proposition 3.5).

- If $b(r)$ admits a finite positive limit $b_{0}$ as $r \rightarrow \infty$, then $\lambda_{n, m}(p) \rightarrow(2 n-1) b_{0}$ for all $m$ as $p \rightarrow-\infty$ (Proposition 3.6).

- If $b(r) \rightarrow \infty$ as $r \rightarrow \infty$, then $\lambda_{n, m}(p) \rightarrow \infty$ as $p \rightarrow-\infty$ (Proposition 3.6)).

Related results concerning the dispersion curves for Schrödinger operator with constant magnetic fields defined on unbounded domains $\Omega \subset \mathbb{R}^{2}$ have been obtained in [5] (the case where $\Omega$ is a strip) and in [4], [7, Section 4.3] (the case where $\Omega$ is a half-plane).

In Theorem 3.8 we formulate the main spectral results which follow from the asymptotic properties of the dispersion curves $\lambda_{n, m}(p), p \in \mathbb{R}$. First, the analyticity and the asymptotics as $p \rightarrow \infty$ of $\lambda_{n, m}(p)$ imply immediately that the spectra $\sigma\left(\mathbf{H}_{m}\right)$ and $\sigma(\mathbf{H})$ of the operators $\mathbf{H}_{m}, m \in \mathbb{Z}$, and $\mathbf{H}$ are purely absolutely continuous. Moreover,

$$
\sigma\left(\mathbf{H}_{m}\right)=\left[\mathcal{E}_{m}, \infty\right), \quad m \in \mathbb{Z}, \quad \sigma(\mathbf{H})=\left[\mathcal{E}_{0}, \infty\right)
$$

where

$$
\mathcal{E}_{m}=\inf _{p \in \mathbb{R}} \lambda_{1, m}(p) \geq 0 .
$$

Next, in the case where the magnetic field tends to 0 as $r \rightarrow \infty$, the spectra of $\mathbf{H}_{m}$, $m \in \mathbb{Z}$, coincide with $[0, \infty)$ and have infinite multiplicity. On the other hand, in the case where the magnetic field tends as $r \rightarrow \infty$ to a positive finite limit, or to infinity, 
we have that $\mathcal{E}_{m}>0$ for all $m \in \mathbb{Z}$ and each of the spectra $\sigma\left(\mathbf{H}_{m}\right)$ contains infinitely many thresholds.

Further, in Section 4, we obtain a convenient formula for the derivatives $\lambda_{n, m}^{\prime}(p)$ which play the role of asymptotic group velocities. Our formula for $\lambda_{n, m}^{\prime}(p)$ yields sufficient conditions (see Theorem 4.3) for positivity of these functions. The leading example when these conditions are met, is

$$
b(r)=b_{0} r^{-\delta}, \quad b_{0}>0, \quad \delta \in[0,1],
$$

and $m \neq 0$. If $\delta=1$, this result remains true for all $m$ (cf. [15]). On the contrary, if $\delta=0$ and $m=0$, then $\lambda_{n, 0}^{\prime}(p)<0$ for all $n$ on some interval of $p$ (lying on the negative halfaxis). Similar results concerning for the dispersion curves for the Schrödinger operator with constant magnetic field, defined on the half-plane with Dirichlet (resp., Neumann) boundary conditions, can be found in [4] (resp., [3] and [7, Section 4.3]).

Finally, in Section 5 we discuss the long-time behaviour of a quantum particle. The time evolution of a quantum system is determined by the unitary groups $\exp \left(-i \mathbf{H}_{m} t\right)$, $m \in \mathbb{Z}$, so that an analysis of its asymptotics as $t \rightarrow \pm \infty$ relies on spectral properties of the operators $\mathbf{H}_{m}$. Since these operators have discrete spectra, a quantum particle remains localized in the $\left(x_{1}, x_{2}\right)$-plane. Its propagation in the $x_{3}$-direction is governed by the group velocities $\lambda_{n, m}^{\prime}(p)$. In particular, the condition $\lambda_{n, m}^{\prime}(p)>0$ for all $n \in \mathbb{N}$ and $p \in \mathbb{R}$ implies that a quantum particle with the magnetic quantum number $m$ propagates as $t \rightarrow+\infty$ in the positive direction of the $x_{3}$-axis.

Let us compare these results with the long-time behaviour of a classical particle in magnetic field (1.5). As shown in [15], the function $x_{3}^{\prime}(t)$ is periodic with period $T$ determined by initial conditions. Its drift $x_{3}(T)-x_{3}(0)$ over the period is nonnegative if $b(r) \geq 0$ and $b^{\prime}(r) \geq 0$. Morever, it is strictly positive if $b^{\prime}(r)>0$ for all $r$. In the case $b(r)=$ const it is still strictly positive if the angular momentum $m$ of a particle is not zero. Thus, our results for functions (1.11) correspond completely to the classical picture if $\delta=1$ or $\delta \in[0,1)$ and $m \neq 0$. In the case $\delta=0$ and $m=0$ the behaviour of quantum and classical particles turn out to be qualitatively different.

\section{HAMILTONIANS AND THEIR DiAgONALIZATIONS}

Here we give precise definitions of the Hamiltonians and discuss their reductions due to the cylindrical symmetry.

2.1. For an arbitrary magnetic potential $A: \mathbb{R}^{3} \rightarrow \mathbb{R}^{3}$ such that $A \in L_{\text {loc }}^{2}\left(\mathbb{R}^{3}\right)^{3}$, the self-adjoint Schrödinger operator (1.2) can be defined via its quadratic form

$$
\mathbf{h}[\mathbf{u}]=\int_{\mathbb{R}^{3}}|i(\nabla \mathbf{u})(x)+A(x) \mathbf{u}(x)|^{2} d x, \quad x=\left(x_{1}, x_{2}, x_{3}\right) .
$$

It is easy to see that this form is closed on the set of functions $u \in L^{2}\left(\mathbb{R}^{3}\right)$ such that $\nabla \mathbf{u} \in L_{\text {loc }}^{1}\left(\mathbb{R}^{3}\right)^{3}$ and $i \nabla \mathbf{u}+A \mathbf{u} \in L^{2}\left(\mathbb{R}^{3}\right)^{3}$. Similarly, if $a \in L_{\text {loc }}^{2}\left(\mathbb{R}^{2}\right)$, then the self-adjoint Schrödinger operator (1.3) can be defined via its quadratic form

$$
h[u ; p]=\int_{\mathbb{R}^{2}}\left(|(\nabla u)(x)|^{2}+(a(x)+p)^{2}|u(x)|^{2}\right) d x, \quad x=\left(x_{1}, x_{2}\right), \quad p \in \mathbb{R} .
$$


This form is closed on the set of functions $u \in L^{2}\left(\mathbb{R}^{2}\right)$ such that integral (2.2) is finite. Clearly, this set does not depend on the parameter $p \in \mathbb{R}$.

Let $\mathcal{F}: L^{2}\left(\mathbb{R}^{3}\right) \rightarrow L^{2}\left(\mathbb{R} ; L^{2}\left(\mathbb{R}^{2}\right)\right)$ be the Fourier transform with respect to $x_{3}$, i.e.

$$
(\mathcal{F} \mathbf{u})\left(x_{1}, x_{2}, p\right)=\frac{1}{\sqrt{2 \pi}} \int_{\mathbb{R}} e^{-i x_{3} p} \mathbf{u}\left(x_{1}, x_{2}, x_{3}\right) d x_{3} .
$$

If $A(x)$ is given by formula (1.1), then

$$
\mathbf{h}[\mathbf{u}]=\int_{-\infty}^{\infty} h[(\mathcal{F} \mathbf{u})(p) ; p] d p
$$

which implies the equation

$$
(\mathcal{F} \mathbf{H u})\left(x_{1}, x_{2} ; p\right)=(H(p) \mathcal{F} \mathbf{u})\left(x_{1}, x_{2} ; p\right) .
$$

This equation can be regarded as a "working" definition of the operator $\mathbf{H}$.

2.2. Assume now that the function $a$ in (1.1) depends only on $r$, and

$$
a \in L_{\mathrm{loc}}^{2}([0, \infty) ; r d r) .
$$

If we separate variables in the cylindrical coordinates $\left(r, \theta, x_{3}\right)$ and denote by $\mathfrak{H}_{m} \subset$ $L_{2}\left(\mathbb{R}^{3}\right)$ the subspace of functions $\mathbf{f}\left(r, x_{3}\right) e^{i m \theta}$ where $\mathbf{f} \in L_{2}\left(\mathbb{R}_{+} \times \mathbb{R} ; r d r d x_{3}\right)$ and $m \in \mathbb{Z}$ is the magnetic quantum number, then

$$
L_{2}\left(\mathbb{R}^{3}\right)=\bigoplus_{m \in \mathbb{Z}} \mathfrak{H}_{m}
$$

The subspaces $\mathfrak{H}_{m}$ are invariant with respect to $\mathbf{H}$ so that restrictions $\mathbf{H}_{m}$ of $\mathbf{H}$ on $\mathfrak{H}_{m}$ are related with $\mathbf{H}$ by formula

$$
\mathbf{H}=\bigoplus_{m \in \mathbb{Z}} \mathbf{H}_{m}
$$

Every $\mathfrak{H}_{m}$ can obviously be identified with the space $L^{2}\left(\mathbb{R}_{+} \times \mathbb{R} ; r d r d x_{3}\right)=: \mathfrak{H}=$; then $\mathbf{H}_{m}$ is identified with operator (1.6).

Quite similarly, if $\mathcal{H}_{m} \subset L_{2}\left(\mathbb{R}^{2}\right)$ is the subspace of functions $f(r) e^{i m \theta}$ where $f \in$ $L_{2}\left(\mathbb{R}_{+} ; r d r\right)$, then

$$
L_{2}\left(\mathbb{R}^{2}\right)=\bigoplus_{m \in \mathbb{Z}} \mathcal{H}_{m}
$$

The subspaces $\mathcal{H}_{m}$ are invariant with respect to $H(p)$ so that restrictions $H_{m}(p)$ of $H(p)$ on $\mathcal{H}_{m}$ are related with $H(p)$ by formula

$$
H(p)=\bigoplus_{m \in \mathbb{Z}} H_{m}(p)
$$

Every $\mathcal{H}_{m}$ can obviously be identified with the space $L^{2}\left(\mathbb{R}_{+} ; r d r\right)=: \mathcal{H}$; then $H_{m}(p)$ is identified with operator (1.4).

Let $\mathcal{F}_{m}: \mathfrak{H}_{m} \rightarrow L^{2}\left(\mathbb{R} ; L^{2}\left(\mathbb{R}_{+} ; r d r\right)\right)$ be the restriction of $\mathcal{F}$ on the subspace $\mathfrak{H}_{m}$. Then we have (cf. (2.3) $)$

$$
\left(\mathcal{F}_{m} \mathbf{H}_{m} \mathbf{f}\right)(r ; p)=\left(H_{m}(p) \mathcal{F}_{m} \mathbf{f}\right)(r ; p)
$$


Sometimes it is more convenient to consider instead of $H_{m}(p)$ the operator

$$
L_{m}(p)=r^{1 / 2} H_{m}(p) r^{-1 / 2}=-\frac{d^{2}}{d r^{2}}+\frac{m^{2}-1 / 4}{r^{2}}+(a(r)+p)^{2}
$$

acting in the space $L^{2}\left(\mathbb{R}_{+}\right)$and unitarily equivalent to the operator $H_{m}(p)$. It is easy to see that the operator $L_{m}(p)$ corresponds to the quadratic form

$$
l_{m}[g ; p]=\int_{0}^{\infty}\left(\left|g^{\prime}(r)\right|^{2}+\left(m^{2}-1 / 4\right) r^{-2}|g(r)|^{2}+(a(r)+p)^{2}|g(r)|^{2}\right) d r,
$$

defined originally on $C_{0}^{\infty}\left(\mathbb{R}_{+}\right)$, and then closed in $L^{2}\left(\mathbb{R}_{+}\right)$.

\subsection{If}

$$
a(r) \rightarrow \infty \quad \text { as } \quad r \rightarrow \infty
$$

then the spectrum of the operator $H_{m}(p), p \in \mathbb{R}, m \in \mathbb{Z}$, is discrete. Thus, it consists of the increasing sequence $\lambda_{n, m}(p)$ of simple eigenvalues. Since $H_{m}(p), p \in \mathbb{R}$, is a Kato analytic family of type (B) (see [9, Chapter VII, Section 4]), all the eigenvalues $\lambda_{n, m}(p)$ are real analytic functions of $p \in \mathbb{R}$. Moreover, $\lambda_{n, m}(p)>0$ because form (2.2) is strictly positive.

In view of formula (2.7) spectral analysis of the operators $\mathbf{H}_{m}$ reduces to a study of a family of functions $\lambda_{n, m}(p), n \in \mathbb{N}$. Indeed, let $\Lambda_{n, m}$ be the operator of multiplication by the function $\lambda_{n, m}(p)$ in the space $L^{2}(\mathbb{R})$. We denote by $\psi_{n, m}(r ; p)$ real normalized eigenfunctions (defined up to signs) of the operators $H_{m}(p)$ and introduce an isometric mapping

by the formula

$$
\Psi_{n, m}: L^{2}(\mathbb{R}) \rightarrow L^{2}\left(\mathbb{R}_{+} \times \mathbb{R} ; r d r d p\right)
$$

Then

$$
\left(\Psi_{n, m} w\right)(p)=\psi_{n, m}(r, p) w(p)
$$

and

$$
L^{2}\left(\mathbb{R}_{+} \times \mathbb{R} ; r d r d p\right)=\bigoplus_{n \in \mathbb{N}} \operatorname{Ran} \Psi_{n, m}
$$

$$
\mathbf{H}_{m}=\bigoplus_{n \in \mathbb{N}} \mathcal{F}_{m}^{*} \Psi_{n, m} \Lambda_{n, m} \Psi_{n, m}^{*} \mathcal{F}_{m}
$$

Together with (2.5), formulas (2.11) and (2.12) justify equations (1.8) for functions (1.7).

\section{Dispersion CURVES AND SPECTRAL ANALYSis}

3.1. In this subsection we consider the operators $H(p)$ acting in the space $L^{2}\left(\mathbb{R}^{2}\right)$ by formula (1.3). Under the assumption $a \in L_{\text {loc }}^{2}\left(\mathbb{R}^{2}\right)$ they are correctly defined by their quadratic forms (2.2). If

$$
a(x) \rightarrow \infty \text { as }|x| \rightarrow \infty, \quad x=\left(x_{1}, x_{2}\right),
$$

then the spectrum of $H(p)$ consists of eigenvalues $\lambda_{n}(p), n \in \mathbb{N}$. We enumerate them in the increasing order with multiplicity taken into account. Our goal is to investigate the asymptotic behaviour of the eigenvalues $\lambda_{n}(p)$ as $p \rightarrow \infty$. Below we denote by $C$ and $c$ different positive constants whose precise values are of no importance. 
We use the following elementary

Lemma 3.1. Let $v(x) \geq 0$. For an arbitrary $\varepsilon>0$, we have the inequality

$$
\int_{\mathbb{R}^{2}} v(x)|u(x)|^{2} d x \leq C \sup _{x \in \mathbb{R}^{2}}\left(\int_{|x-y| \leq \varepsilon} v^{2}(y) d y\right)^{1 / 2} \int_{\mathbb{R}^{2}}\left(\varepsilon|\nabla u(x)|^{2}+\varepsilon^{-1}|u(x)|^{2}\right) d x
$$

provided the supremum in the right-hand side is finite.

Proof. Let $\Pi_{\varepsilon} \subset \mathbb{R}^{2}$ be a square of length $\varepsilon$. We proceed from the estimate

$$
\left(\int_{\Pi_{\varepsilon}}|u(x)|^{4} d x\right)^{1 / 2} \leq C\left(\varepsilon \int_{\Pi_{\varepsilon}}|\nabla u(x)|^{2} d x+\varepsilon^{-1} \int_{\Pi_{\varepsilon}}|u(x)|^{2} d x\right)
$$

which follows from the Sobolev embedding theorem by a scaling transformation. Using the Schwarz inequality, we deduce from this estimate that

$$
\int_{\Pi_{\varepsilon}} v(x)|u(x)|^{2} d x \leq C\left(\int_{\Pi_{\varepsilon}} v^{2}(x) d x\right)^{1 / 2}\left(\varepsilon \int_{\Pi_{\varepsilon}}|\nabla u(x)|^{2} d x+\varepsilon^{-1} \int_{\Pi_{\varepsilon}}|u(x)|^{2} d x\right) .
$$

Let us split the space $\mathbb{R}^{2}$ in the lattice of squares $\Pi_{\varepsilon}^{(n)}$ of length $\varepsilon$. Applying (3.3) to every $\Pi_{\varepsilon}^{(n)}$ and summing over all $n$, we arrive at (3.2).

In the following assertion we do not assume (3.1).

Proposition 3.2. Let $a \in L_{\text {loc }}^{2}\left(\mathbb{R}^{2}\right)$. Set $a_{-}(x)=\max \{-a(x), 0\}$,

$$
\alpha(\varepsilon)=\sup _{x \in \mathbb{R}^{2}} \int_{|x-y| \leq \varepsilon} a_{-}^{2}(y) d y
$$

and suppose that $\alpha(\varepsilon) \rightarrow 0$ as $\varepsilon \rightarrow 0$. Then we have

$$
\liminf _{p \rightarrow \infty} p^{-2} \inf \sigma(H(p)) \geq 1
$$

Proof. Applying estimate (3.2) with $\varepsilon=p^{-1}$ to the function $v=a_{-}$, we find that

$$
\begin{gathered}
\int_{\mathbb{R}^{2}}\left(|\nabla u|^{2}+(p+a)^{2}\right)|u|^{2} d x \geq \int_{\mathbb{R}^{2}}\left(|\nabla u|^{2}+\left(-2 p a_{-}+p^{2}\right)|u|^{2}\right) d x \\
\geq \int_{\mathbb{R}^{2}}\left(|\nabla u|^{2}+p^{2}\right)|u|^{2} d x-C \sqrt{\alpha\left(p^{-1}\right)} \int_{\mathbb{R}^{2}}\left(|\nabla u|^{2}+p^{2}\right)|u|^{2} d x .
\end{gathered}
$$

Since $\alpha\left(p^{-1}\right) \rightarrow 0$ as $p \rightarrow \infty$, this implies (3.4).

Proposition 3.3. Let $a \in L_{\text {loc }}^{2}\left(\mathbb{R}^{2}\right)$ and let condition (3.1) be satisfied. Then, for all $n \in \mathbb{N}$, we have

$$
\lambda_{n}(p)=p^{2}(1+o(1)), \quad p \rightarrow \infty .
$$

Proof. Under condition (3.1) the function $a_{-}$has compact support so that we can use Proposition 3.2 and estimate (3.4) implies

$$
\liminf _{p \rightarrow \infty} p^{-2} \lambda_{n}(p) \geq 1
$$


Set $G(\varepsilon)=-\Delta+\left(1+\varepsilon^{-1}\right) a^{2}(x), \varepsilon>0$. The spectrum of $G(\varepsilon)$ is discrete; let $\nu_{n}, n \in \mathbb{N}$, be the increasing sequence of its eigenvalues. By the elementary inequality

$$
(a+p)^{2} \leq\left(1+\varepsilon^{-1}\right) a^{2}+(1+\varepsilon) p^{2}, \quad \varepsilon>0,
$$

we have $H(p) \leq G(\varepsilon)+(1+\varepsilon) p^{2}$ so that by the minimax principle

$$
\lambda_{n}(p) \leq \nu_{n}(\varepsilon)+(1+\varepsilon) p^{2} .
$$

Therefore, for all $\varepsilon>0$,

$$
\limsup _{p \rightarrow \infty} p^{-2} \lambda_{n}(p) \leq 1+\varepsilon,
$$

which combined with (3.6) yields (3.5).

Corollary 3.4. Suppose that the function a depends on $r$ only. Let conditions (2.4) and (2.10) be satisfied. Then, for all $n \in \mathbb{N}, m \in \mathbb{Z}$, we have

$$
\lambda_{n, m}(p)=p^{2}(1+o(1)), \quad p \rightarrow \infty .
$$

3.2. From now on we always assume that the function a depends on $r$ only and that conditions (2.4) and (2.10) are satisfied. In this subsection we investigate the asymptotics as $p \rightarrow-\infty$ of the eigenvalues $\lambda_{n, m}(p)$ of the operators $H_{m}(p)$. Actually, it is more convenient to work with the operators $L_{m}(p)$ acting in the space $L^{2}\left(\mathbb{R}_{+}\right)$by formula (2.8). We suppose that the function $a$ is differentiable at least for suffiently big $r$ and formulate the results in terms of the function $b(r)=a^{\prime}(r)$ related to the magnetic field by formula (1.5).

Remark first that if $k=-p>0$ is big enough, then the equation

$$
a(r)=k
$$

has at least one solution. We denote by $\rho_{k}$ the greatest solution of (3.7). Clearly, $\rho_{k} \rightarrow \infty$ as $k \rightarrow \infty$.

\section{Proposition 3.5. Suppose that}

$$
\lim _{r \rightarrow \infty} b(r)=0 .
$$

Then for each $n \in \mathbb{N}$ and $m \in \mathbb{Z}$ we have

$$
\lim _{k \rightarrow \infty} \lambda_{n, m}(-k)=0 .
$$

Proof. Set

$$
\mathbf{b}(r)=\sup _{x \geq r}|b(x)| \quad \text { and } \quad \gamma_{k}=\mathbf{b}\left(\rho_{k}\right)^{-1 / 2} .
$$

Let us fix $n \in \mathbb{N}$. We pick a function $\phi_{1} \in C_{0}^{\infty}(\mathbb{R})$ such that $\operatorname{supp} \phi_{1}=\left[0, \frac{1}{2 n}\right]$ and, for $n>1$, set

$$
\phi_{j}(x)=\phi_{1}(x-(j-1) / n), \quad x \in \mathbb{R}, \quad j=2, \ldots, n .
$$

For $k>0$ large enough, we put

$$
\varphi_{j}(r ; k)=\gamma_{k}^{-1 / 2} \phi_{j}\left(\frac{r-\rho_{k}}{\gamma_{k}}\right), \quad r \geq 0, \quad j=1, \ldots, n .
$$


We will prove now that for quadratic form (2.9)

$$
\lim _{k \rightarrow \infty} l_{m}\left[\varphi_{j}(k) ;-k\right]=0 \text {. }
$$

It follows from (3.11) that

$$
\int_{0}^{\infty}\left|\varphi_{j}^{\prime}(r ; k)\right|^{2} d r \leq C \gamma_{k}^{-2}
$$

with $C$ independent of $k$. Further, since $\operatorname{supp} \varphi_{j}(k) \subset\left[\rho_{k}, \rho_{k}+\gamma_{k}\right]$, we have

$$
\int_{0}^{\infty} r^{-2}\left|\varphi_{j}(r ; k)\right|^{2} d r \leq C \rho_{k}^{-2}
$$

Similarly,

$$
\int_{0}^{\infty}(a(r)-k)^{2}\left|\varphi_{j}(r ; k)\right|^{2} d r \leq C \sup _{r \in\left(\rho_{k}, \rho_{k}+\gamma_{k}\right)}(a(r)-k)^{2} .
$$

Using the condition $a\left(\rho_{k}\right)=k$, we obtain, for $r \geq \rho_{k}$, the bound

$$
(a(r)-k)^{2}=\left(a(r)-a\left(\rho_{k}\right)\right)^{2}=\left(\int_{\rho_{k}}^{r} b(s) d s\right)^{2} \leq\left(r-\rho_{k}\right)^{2} \mathbf{b}^{2}\left(\rho_{k}\right)
$$

where $\mathbf{b}$ is function (3.10). Thus, the right-hand side in (3.15) is bounded by $C \gamma_{k}^{2} \mathbf{b}^{2}\left(\rho_{k}\right)$. Putting together this result with inequalities (3.13), (3.14) and taking into account (3.10), we get

$$
l_{m}\left[\varphi_{j}(k) ;-k\right] \leq C\left(\mathbf{b}\left(\rho_{k}\right)+\rho_{k}^{-2}\right) .
$$

This yields (3.12).

Let us use now that the supports of the functions $\varphi_{j}(k), j=1, \ldots, n$, are disjoint and set

$$
\mathcal{L}_{n}(k)=\operatorname{span}\left\{\varphi_{1}(k), \ldots, \varphi_{n}(k)\right\} .
$$

Then $\operatorname{dim} \mathcal{L}_{n}(k)=n$ and according to (3.12) $l_{m}[\varphi(k) ;-k] \rightarrow 0$ as $k \rightarrow \infty$ for all $\varphi(k) \in \mathcal{L}_{n}(k)$ with $\|\varphi(k)\|=1$. By the mini-max principle this implies (3.9)).

The proof of Proposition 3.6 relies on a comparison of the operator $L_{m}(-k)$ with the "model" operator

$$
T(k)=-\frac{d^{2}}{d x^{2}}+b^{2}\left(\varrho_{k}\right)\left(x-\varrho_{k}\right)^{2}, \quad x \in \mathbb{R},
$$

acting in the space $L^{2}(\mathbb{R})$. Let $f_{j}$ be the normalized in $L^{2}(\mathbb{R})$ real-valued eigenfunctions (defined up to sign) of the harmonic oscillator, i.e.

$$
-f_{j}^{\prime \prime}(x)+x^{2} f_{j}(x)=(2 j-1) f_{j}(x), \quad x \in \mathbb{R}, \quad j \in \mathbb{N} .
$$

Then

$$
\psi_{j}(x ; k)=b\left(\varrho_{k}\right)^{1 / 4} f_{j}\left(b\left(\varrho_{k}\right)^{1 / 2}\left(x-\varrho_{k}\right)\right)
$$

are normalized eigenfunctions of the operator $T(k)$, that is

$$
T(k) \psi_{j}(k)=b\left(\varrho_{k}\right)(2 j-1) \psi_{j}(k), \quad j \in \mathbb{N} .
$$

The proof of the following result follows the general lines of the proof of $[2$, Theorem 11.1]. 
Proposition 3.6. Suppose that a $(r)$ is locally semibounded from above. For $r>0$ large enough, we assume that the function $b(r)$ is differentiable and that conditions

$$
\begin{gathered}
b(r)>0, \\
\lim _{r \rightarrow \infty} r^{2} b(r)=\infty,
\end{gathered}
$$

as well as

$$
\lim _{r \rightarrow \infty} b(r)^{-3} \mathbf{b}_{1}^{2}(r)=0, \quad \text { where } \quad \mathbf{b}_{1}(r)=\sup _{r / 2 \leq x \leq 3 r / 2}\left|b^{\prime}(x)\right|,
$$

are satisfied. Let also

$$
\lim _{k \rightarrow \infty} k^{-2} b\left(\rho_{k}\right)=0 .
$$

Then, for all $n \in \mathbb{N}, m \in \mathbb{Z}$, we have

$$
\lambda_{n, m}(-k)=b\left(\varrho_{k}\right)(2 n-1+o(1)), \quad k \rightarrow \infty .
$$

Proof. Due to the minimax principle, it suffices to show that:

(i) For each $n \in \mathbb{N}$ and sufficiently large $k$ there exists a subspace $\mathcal{L}_{n}(k)$ of $L^{2}\left(\mathbb{R}_{+}\right)$such that $\operatorname{dim} \mathcal{L}_{n}(k)=n, \mathcal{L}_{n}(k) \subset D\left(L_{m}(-k)\right)$, and for each $\varphi(k) \in \mathcal{L}_{n}(k)$ we have

$$
\left\langle L_{m}(-k) \varphi(k), \varphi(k)\right\rangle \leq b\left(\varrho_{k}\right)(2 n-1+o(1))\|\varphi(k)\|^{2}, \quad k \rightarrow \infty .
$$

(ii) For each $n \in \mathbb{N}$ there exists a bounded operator $R_{n}(k)$ such that $\operatorname{rank} R_{n}(k) \leq n-1$ (hence, $R_{1}(k)=0$ ), and

$$
L_{m}(-k) \geq b\left(\varrho_{k}\right)(2 n-1+o(1)) I+R_{n}(k), \quad k \rightarrow \infty .
$$

We pick $\gamma_{k}>0$ such that

$$
\begin{gathered}
\gamma_{k} \rightarrow 0, \\
\gamma_{k} \varrho_{k} b\left(\varrho_{k}\right)^{1 / 2} \rightarrow \infty, \\
\gamma_{k}^{-3} b\left(\varrho_{k}\right)^{-3 / 2} \mathbf{b}_{1}\left(\varrho_{k}\right) \rightarrow 0
\end{gathered}
$$

as $k \rightarrow \infty$. Note that (3.29) is compatible with (3.28) due to (3.22), and (3.30) is compatible with (3.28) due to (3.23).

Proof of (i). Let $\zeta \in C_{0}^{\infty}(\mathbb{R})$ be such that $0 \leq \zeta(x) \leq 1, \zeta(x)=1$ for $|x| \leq 1 / 2$ and $\operatorname{supp} \zeta=[-1,1]$. For $k$ large enough, set

$$
\zeta(r ; k)=\zeta\left(\gamma_{k} b\left(\varrho_{k}\right)^{1 / 2}\left(r-\varrho_{k}\right)\right), \quad r \in \mathbb{R}_{+},
$$

and

$$
\varphi_{j}(r ; k)=\psi_{j}(r ; k) \zeta(r ; k), \quad r \in \mathbb{R}_{+}, \quad j \in \mathbb{N},
$$

the functions $\psi_{j}(r ; k)$ being defined in (3.19). It follows from (3.29) that

$$
\operatorname{supp} \varphi_{j}(k)=\left[\varrho_{k}-\gamma_{k}^{-1} b\left(\varrho_{k}\right)^{-1 / 2}, \varrho_{k}+\gamma_{k}^{-1} b\left(\varrho_{k}\right)^{-1 / 2}\right] \subset\left[\varrho_{k} / 2,3 \varrho_{k} / 2\right]
$$

and, in particular, $\varphi_{j}(k) \in D\left(L_{m}(-k)\right)$. Note that

$$
\left\langle\varphi_{j}(k), \varphi_{l}(k)\right\rangle_{L^{2}\left(\mathbb{R}_{+}\right)}=\delta_{j l}-\int_{\mathbb{R}} \psi_{j}(x ; k) \psi_{l}(x ; k)\left(1-\zeta^{2}(x ; k)\right) d x=\delta_{j l}+o(1)
$$


as $k \rightarrow \infty$. Indeed, the integral here can be estimated by

$$
\int_{\mathbb{R}}\left|f_{j}(x) f_{l}(x)\right|\left(1-\zeta^{2}\left(\gamma_{k} x\right)\right) d x \leq \int_{|x| \geq\left(2 \gamma_{k}\right)^{-1}}\left|f_{j}(x) f_{l}(x)\right| d x
$$

which tends to zero according to (3.28). In particular, 3.33 implies that for all $n \in \mathbb{N}$ the functions $\varphi_{1}(k), \ldots, \varphi_{n}(k)$ are linearly independent if $k$ is large enough. Thus, the space $\mathcal{L}_{n}(k)$ defined by (3.16) ) has dimension $n$.

Let us set

$$
\psi(x ; k)=\sum_{j=1}^{n} c_{j} \psi_{j}(x ; k), \quad c_{j} \in \mathbb{C},
$$

$\varphi(r ; k)=\psi(r ; k) \zeta(r ; k)$ and consider $\left\langle L_{m}(-k) \varphi(k), \varphi(k)\right\rangle$. Integrating by parts, we find that

$$
-2 \operatorname{Re}\left\langle\psi^{\prime}(k) \zeta^{\prime}(k), \psi(k) \zeta(k)\right\rangle-\left\langle\psi(k) \zeta^{\prime \prime}(k), \psi(k) \zeta(k)\right\rangle=\left\|\psi(k) \zeta^{\prime}(k)\right\|^{2}
$$

so that

$$
\begin{aligned}
\left\langle L_{m}(-k) \varphi(k), \varphi(k)\right\rangle & =\operatorname{Re}\left\langle-\psi^{\prime \prime}(k)+(a(r)-k)^{2} \psi(k), \psi(k) \zeta^{2}(k)\right\rangle \\
& +\left\|\psi(k) \zeta^{\prime}(k)\right\|^{2}+\left(m^{2}-1 / 4\right)\left\|r^{-1} \varphi(k)\right\|^{2} .
\end{aligned}
$$

We assume that $\|\varphi(k)\|=1$ and hence according to (3.33) $\|\psi(k)\|=1+o(1)$. The second and third terms in the right-hand side of (3.35) are negligible. Indeed, differentiating (3.31) and using condition (3.28), we find that

$$
\left\|\psi(k) \zeta^{\prime}(k)\right\|^{2}=O\left(b\left(\varrho_{k}\right) \gamma_{k}^{2}\right)=o\left(b\left(\varrho_{k}\right)\right) .
$$

Since $r^{-1} \leq 2 \varrho_{k}^{-1}$ on the support of $\varphi(k)$, relation (3.22) implies

$$
\left\|r^{-1} \varphi(k)\right\|^{2}=O\left(\varrho_{k}^{-2}\right)=o\left(b\left(\varrho_{k}\right)\right), \quad k \rightarrow \infty .
$$

Further we consider the first term in the right-hand side of (3.35). It follows from equation (3.20) that

$$
-\psi_{j}^{\prime \prime}(k)+(a(r)-k)^{2} \psi_{j}(k)=b\left(\varrho_{k}\right)(2 j-1) \psi_{j}(k)+\alpha(k) \psi_{j}(k)
$$

where the function

$$
\alpha(r ; k)=(a(r)-k)^{2}-b^{2}\left(\varrho_{k}\right)\left(r-\varrho_{k}\right)^{2} .
$$

Let us estimate the right-hand side. In view of the equation $a\left(\rho_{k}\right)=k$, a second-order Taylor expansion of $a$ at $\varrho_{k}$ yields

$$
a(r)=k+b\left(\varrho_{k}\right)\left(r-\varrho_{k}\right)+\int_{\varrho_{k}}^{r} b^{\prime}(s)(r-s) d s .
$$

Therefore,

$$
\alpha(r ; k)=2 b\left(\varrho_{k}\right)\left(r-\varrho_{k}\right) \int_{\varrho_{k}}^{r} b^{\prime}(s)(r-s) d s+\left(\int_{\varrho_{k}}^{r} b^{\prime}(s)(r-s) d s\right)^{2},
$$

and hence

$$
\begin{gathered}
|\alpha(r ; k)| \leq b\left(\varrho_{k}\right) \mathbf{b}_{1}\left(\varrho_{k}\right)\left|r-\varrho_{k}\right|^{3}+4^{-1} \mathbf{b}_{1}^{2}\left(\varrho_{k}\right)\left(r-\varrho_{k}\right)^{4} \\
\quad \leq \gamma_{k}^{-3} b\left(\varrho_{k}\right)^{-1 / 2} \mathbf{b}_{1}\left(\varrho_{k}\right)+4^{-1} \gamma_{k}^{-4} b\left(\varrho_{k}\right)^{-2} \mathbf{b}_{1}^{2}\left(\varrho_{k}\right)
\end{gathered}
$$


provided that $\left|r-\varrho_{k}\right| \leq \gamma_{k}^{-1} b\left(\varrho_{k}\right)^{-1 / 2}$. In view of conditions (3.28) and (3.30), this gives us the estimate

so that

$$
\sup _{\left|r-\varrho_{k}\right| \leq \gamma_{k}^{-1} b\left(\varrho_{k}\right)^{-1 / 2}}|\alpha(r ; k)|=o\left(b\left(\rho_{k}\right)\right)
$$

$$
\left(-\psi_{j}^{\prime \prime}(k)+(a(r)-k)^{2} \psi_{j}(k)\right) \zeta(k)=b\left(\varrho_{k}\right)(2 j-1) \varphi_{j}(k)+o\left(b\left(\rho_{k}\right)\right) .
$$

Thus, using also (3.33) we obtain that

$$
\begin{aligned}
\operatorname{Re}\left\langle-\psi^{\prime \prime}(k)+(a(r)-k)^{2} \psi(k), \psi(k) \zeta^{2}(k)\right\rangle & =b\left(\varrho_{k}\right) \sum_{j, l=1}^{n}(2 j-1) c_{j} \bar{c}_{l}\left\langle\varphi_{j}(k), \varphi_{l}(k)\right\rangle+o\left(b\left(\rho_{k}\right)\right) \\
& \leq b\left(\varrho_{k}\right)(2 n-1)+o\left(b\left(\rho_{k}\right)\right) .
\end{aligned}
$$

Together with (3.36) and (3.37), this implies estimate (3.26) for each $\varphi(k) \in \mathcal{L}_{n}(k)$.

Proof of (ii). Let functions $\zeta \in C_{0}^{\infty}(\mathbb{R})$ and $\eta \in C^{\infty}(\mathbb{R})$ satisfy $\zeta^{2}(x)+\eta^{2}(x)=1$, $x \in \mathbb{R}$; moreover, as before, we require that $0 \leq \zeta(x) \leq 1, \zeta(x)=1$ for $|x| \leq 1 / 2$ and $\operatorname{supp} \zeta=[-1,1]$. By analogy with (3.31) set

$$
\eta(r ; k)=\eta\left(\gamma_{k} b\left(\varrho_{k}\right)^{1 / 2}\left(r-\varrho_{k}\right)\right), \quad r \in \mathbb{R}_{+} .
$$

Then we have

$$
\zeta^{2}(r ; k)+\eta^{2}(r ; k)=1, \quad r \in \mathbb{R}_{+} .
$$

We proceed from the localization formula (known as the IMS formula - see e.g. [2, Section 3.1])

$$
L_{m}(-k)=\zeta(k) L_{m}(-k) \zeta(k)+\eta(k) L_{m}(-k) \eta(k)-\zeta^{\prime}(k)^{2}-\eta^{\prime}(k)^{2},
$$

where $\zeta(k), \eta(k), \zeta^{\prime}(k)$ and $\eta^{\prime}(k)$ are understood as operators of multiplication by the functions $\zeta(r, k), \eta(r, k), \zeta^{\prime}(r, k)$ and $\eta^{\prime}(r, k)$, respectively. According to (3.28) it follows from definitions (3.31) and (3.42) that

$$
\max _{r \in \mathbb{R}_{+}}\left(\zeta^{\prime}(r, k)^{2}+\eta^{\prime}(r, k)^{2}\right)=O\left(\gamma_{k}^{2} b\left(\varrho_{k}\right)\right)=o\left(b\left(\varrho_{k}\right)\right), \quad k \rightarrow \infty .
$$

Next, we check that

$$
\eta(k) L_{m}(-k) \eta(k) \geq \nu_{k} b\left(\varrho_{k}\right) \eta^{2}(k)
$$

with $\nu_{k} \rightarrow \infty$ as $k \rightarrow \infty$. By virtue of the Hardy inequality

$$
\eta(k)\left(-\frac{d^{2}}{d r^{2}}+\frac{m^{2}-1 / 4}{r^{2}}\right) \eta(k) \geq 0,
$$

it suffices to check that

for

$$
(a(r)-k)^{2} \geq \nu_{k} b\left(\varrho_{k}\right)
$$

$$
r \geq \varrho_{k}+\left(2 \gamma_{k} b\left(\varrho_{k}\right)^{1 / 2}\right)^{-1}=: \varrho_{k}^{(+)} \quad \text { and } \quad r \leq \varrho_{k}-\left(2 \gamma_{k} b\left(\varrho_{k}\right)^{1 / 2}\right)^{-1}=: \varrho_{k}^{(-)} .
$$

According to (3.21) there exists $r_{0}$ such that the function $a(r)$ is increasing for $r \geq r_{0}$.

Let first $r \geq r_{0}$. Then

$$
|a(r)-k|=\left|a(r)-a\left(\varrho_{k}\right)\right| \geq \pm\left(a\left(\varrho_{k}^{( \pm)}\right)-a\left(\varrho_{k}\right)\right)
$$


if $\pm\left(r-\varrho_{k}^{( \pm)}\right) \geq 0$ and $r \geq r_{0}$. It follows from definition (3.46) of the numbers $\varrho_{k}^{( \pm)}$that

$$
a\left(\varrho_{k}^{( \pm)}\right)-a\left(\varrho_{k}\right)=\int_{\varrho_{k}}^{\varrho_{k}^{( \pm)}} b(s) d s= \pm\left(2 \gamma_{k}\right)^{-1} b\left(\varrho_{k}\right)^{1 / 2}+\int_{\varrho_{k}}^{\varrho_{k}^{( \pm)}}\left(b(s)-b\left(\varrho_{k}\right)\right) d s .
$$

The absolute value of the integral in the right-hand side can be estimated by

$$
\left|\int_{\varrho_{k}}^{\varrho_{k}^{( \pm)}} d s \int_{\varrho_{k}}^{s}\right| b^{\prime}(\sigma)|d \sigma| \leq 2^{-1} \mathbf{b}_{1}\left(\rho_{k}\right)\left(\varrho_{k}^{( \pm)}-\varrho_{k}\right)^{2}=8^{-1} \mathbf{b}_{1}\left(\rho_{k}\right) \gamma_{k}^{-2} b\left(\varrho_{k}\right)^{-1}
$$

where the function $\mathbf{b}_{1}$ is defined in (3.23). By virtue of conditions (3.28) and (3.30) this expression is $o\left(\gamma_{k}^{-1} b\left(\varrho_{k}\right)^{1 / 2}\right)$ as $k \rightarrow \infty$. Therefore the absolute value of expression (3.48) is bounded from below by $\left(3 \gamma_{k}\right)^{-1} b\left(\varrho_{k}\right)^{1 / 2}$. Thus, for $r \geq r_{0}$, estimate (3.45) with $\nu_{k}=\left(3 \gamma_{k}\right)^{-2} \rightarrow \infty$ is a consequence of (3.47).

If $r \leq r_{0}$, we take into account that $a(r)$ is semibounded from above so that $(a(r)-$ $k)^{2} \geq 2^{-1} k^{2}$. Hence estimate (3.47) with $\nu_{k}=2^{-1} k^{2} b\left(\varrho_{k}\right)^{-1} \rightarrow \infty$ is satisfied according to condition (3.24).

Putting together definitions (2.8) and (3.17) of the operators $L_{m}(-k)$ and $T(k)$, we see that

$$
\zeta(k) L_{m}(-k) \zeta(k)=\zeta(k) T(k) \zeta(k)+\alpha(k) \zeta^{2}(k),
$$

where $\alpha(k)$ is the operator of multiplication by function (3.39). The first term in the right-hand side is bounded from below by $b\left(\varrho_{k}\right) \zeta^{2}(k)$ because $b\left(\varrho_{k}\right)$ is the first eigenvalue of the operator $T(k)$. By virtue of (3.40) the second term satisfies the estimate

$$
\left\|\alpha(k) \zeta^{2}(k)\right\|=o\left(b\left(\varrho_{k}\right)\right) .
$$

It follows that operator (3.49) is bounded from below by $b\left(\varrho_{k}\right) \zeta^{2}(k)-o\left(b\left(\varrho_{k}\right)\right) I$. Combining this result with (3.43) and (3.44), we get estimate (3.27) in the case $n=1$.

If $n \geq 2$, we denote by $P_{n}(k)$ the orthogonal projection onto the span of the first $n-1$ eigenfunctions of the operator $T(k)$. Then $T(k)\left(I-P_{n}(k)\right) \geq(2 n-1)\left(I-P_{n}(k)\right)$ and hence

$$
\begin{gathered}
\zeta(k) T(k) \zeta(k)=\zeta(k) T(k)\left(I-P_{n}(k)\right) \zeta(k)+\zeta(k) T(k) P_{n}(k) \zeta(k) \\
\geq b\left(\varrho_{k}\right)(2 n-1) \zeta(k)\left(I-P_{n}(k)\right) \zeta(k)+\zeta(k) T(k) P_{n}(k) \zeta(k)=b\left(\varrho_{k}\right)(2 n-1) \zeta^{2}(k)+R_{n}(k)
\end{gathered}
$$

where

$$
R_{n}(k)=\zeta(k)\left(T(k)-b\left(\varrho_{k}\right)(2 n-1) I\right) P_{n}(k) \zeta(k) .
$$

Clearly, rank $R_{n}(k) \leq n-1$. Putting together (3.43), (3.44) and (3.49) - (3.51), we obtain (3.27) in the case $n \geq 2$.

Example 3.7. Let $b(r)=b_{0} r^{-\delta}, b_{0}>0, \delta \leq 1$, for sufficiently large $r$. Then $\mathbf{b}_{1}(r)=$ $b_{0} \delta r^{-\delta-1}$ and conditions (3.21) $-(3.23)$ are satisfied. Moreover, $\rho_{k}=c_{1} k^{\nu}$ and $k^{2} b\left(\rho_{k}\right)=$ $c_{2} k^{-1-\nu}$ where $\nu=(1-\delta)^{-1}$ and $c_{1}, c_{2}>0$ if $\delta<1$. If $\delta=1$, then $\rho_{k}=\exp \left(b_{0}^{-1} k\right)$ and $k^{2} b\left(\rho_{k}\right)=k^{2} b_{0} \exp \left(-b_{0}^{-1} k\right)$. In both cases condition (3.24) is also satisfied. Thus, Proposition [3.6 implies the following results. If $\delta>0$, then $\lambda_{n, m}(p) \rightarrow 0$ as $p \rightarrow-\infty$ (this result follows also from Proposition 3.5). If $\delta=0$, then the functions $\lambda_{n, m}(p)$ have 
finite limits $b_{0}(2 n-1)$ as $p \rightarrow-\infty$. If $\delta<0$, then these functions tend to $+\infty$ as $p \rightarrow-\infty$.

3.3. Let us return to the Hamiltonians $\mathbf{H}_{m}$ and $\mathbf{H}$ defined in Section 2 .

Theorem 3.8. Assume (2.4) and (2.10).

(i) Then all operators $\mathbf{H}_{m}, m \in \mathbb{Z}$, and hence $\mathbf{H}$ are absolutely continuous and their spectra coincide with the half-axes defined by equations (1.9) and (1.10).

(ii) If the hypotheses of Proposition 3.5 hold true, then $\mathcal{E}_{m}=0$ for all $m \in \mathbb{Z}$. Moreover, the multiplicities of all spectra $\sigma\left(\mathbf{H}_{m}\right)$ and hence of $\sigma(\mathbf{H})$ are infinite.

(iii) Let the hypotheses of Proposition 3.6 hold true. If $b(r) \rightarrow \infty$, then the infimum in (1.10) is attained (at a finite point) so that for all $m \in \mathbb{Z}$

$$
\mathcal{E}_{m}=\min _{p \in \mathbb{R}} \lambda_{1, m}(p)>0 .
$$

(iv) Let the hypotheses of Proposition 3.6 hold true. If b(r) admits a finite positive limit $b_{0}$ as $r \rightarrow \infty$, then $\mathcal{E}_{m} \in\left(0, b_{0}\right]$ for all $m \in \mathbb{Z}$.

Proof. It suffices to prove only the assertions concerning the operators $\mathbf{H}_{m}$. In view of decomposition (2.12) they reduce to corresponding statements about the operators $\Lambda_{n, m}$. These operators are absolutely continuous because the eigenvalues $\lambda_{n, m}(p)$ are real analytic functions of $p \in \mathbb{R}$ which are non constants since according to Corollary 3.4 $\lambda_{n, m}(p) \rightarrow \infty$ as $p \rightarrow \infty$. Moreover, we have that

$$
\sigma\left(\Lambda_{n, m}\right)=\left[\mathcal{E}_{n, m}, \infty\right) \quad \text { where } \quad \mathcal{E}_{n, m}=\inf _{p \in \mathbb{R}} \lambda_{n, m}(p) \geq 0
$$

because $\lambda_{n, m}(p)>0$ for all $p \in \mathbb{R}$. This implies relations (1.9) with $\mathcal{E}_{m}$ defined by (1.10).

In case (ii) it suffices to use that according to (3.9) $\mathcal{E}_{n, m}=0$ and hence $\sigma\left(\Lambda_{n, m}\right)=$ $[0, \infty)$ for all $m$ and $n$.

In case (iii) Proposition 3.6 implies that $\lambda_{n, m}(p) \rightarrow \infty$ as $p \rightarrow-\infty$ for all $n$ and $m$ so that

$$
\mathcal{E}_{n, m}=\min _{p \in \mathbb{R}} \lambda_{n, m}(p)>0
$$

and hence infimum in (1.10) can be replaced by minimum.

In case (iv) we use that according to (3.25) $\mathcal{E}_{n, m} \leq(2 n-1) b_{0}$. Moreover, $\mathcal{E}_{n, m}>0$ because $\lambda_{n, m}(p)>0$ for all $p \in \mathbb{R}$. For $n=1$, this gives the desired result.

Remark 3.9. According to (2.12) and (3.52) the spectrum of the operator $\mathbf{H}_{m}$ consists of the "branches" $\left[\mathcal{E}_{n, m}, \infty\right)$ where the points $\mathcal{E}_{n, m}$ are called thresholds. In cases (iii) and (iv)

$$
\mathcal{E}_{n, m}<\mathcal{E}_{n+1, m}
$$

for all $n \in \mathbb{N}$. Indeed, in case iii) (3.54) is a consequence of the estimate $\lambda_{n, m}(p)<$ $\lambda_{n+1, m}(p)$ valid for all $p \in \mathbb{R}$ and of formula (3.53). In case (iv) one has to take additionally into account that the limit of $\lambda_{n, m}(p)$ as $p \rightarrow-\infty$ is strictly smaller than that of $\lambda_{n+1, m}(p)$. Inequality (3.54) means that there are infinitely many distinct thresholds in each of the spectra $\sigma\left(\mathbf{H}_{m}\right), m \in \mathbb{Z}$, and hence in $\sigma(\mathbf{H})$. 
Remark 3.10. In case( iii) the multiplicity of the spectrum of all operators $\Lambda_{n, m}$ equals at least to 2 whereas in cases (ii) and (iv) it might be equal to 1 .

\section{Group velocities}

4.1. In this subsection we obtain a formula for the derivative $\lambda_{n, m}^{\prime}(p), n \in \mathbb{N}, m \in \mathbb{Z}$, which yields sufficient conditions for the monotonicity of $\lambda_{n, m}(p)$ as a function of $p$. Recall that the operators $H_{m}(p), m \in \mathbb{N}, p \in \mathbb{R}$, were defined in the space $\mathcal{H}$ by formula (1.4).

The proof of Theorem 4.3 relies on integration by parts. To prove that non-integral terms disappear at $r=0$, we use standard bounds on $\psi_{n, m}(r ; p)$. Unfortunately, we were unable to find necessary results in the literature and therefore give their brief proofs.

Let us consider the differential equation of Bessel type

$$
-r^{-1}\left(r y^{\prime}\right)^{\prime}+m^{2} r^{-2} y+q(r) y=0, \quad m=0,1,2, \ldots,
$$

in a neighborhood $\left(0, r_{0}\right)$ of the point $r=0$. If $q(r)=0$, then it has the regular $y_{0}^{(r e g)}(r)=r^{m}$ and singular $y_{0}^{(\text {sing })}(r)=r^{-m}$ solutions for $m \neq 0$ and $y_{0}^{(r e g)}(r)=1$ and $y_{0}^{(\operatorname{sing})}(r)=\ln r$ for $m=0$.

Lemma 4.1. Let $m \neq 0$, and let the function $r q(r)$ belong to the class $L^{1}\left(0, r_{0}\right)$. Then equation (4.1) has a solution $y^{(r e g)}(r)$ satisfying the relation

$$
y^{(r e g)}(r)=r^{m}+o\left(r^{m}\right), \quad r \rightarrow 0 .
$$

For its derivative, we have the bound

$$
d y^{(r e g)}(r) / d r=O\left(r^{m-1}\right)
$$

Let $m=0$. Suppose that the function $r \ln r q(r)$ belongs to the class $L^{1}\left(0, r_{0}\right)$. Then equation (4.1) has a solution $y^{(r e g)}(r)$ satisfying relation (4.2) where $m=0$. For its derivative, we have the bound

$$
d y^{(r e g)}(r) / d r=O\left(\int_{0}^{r}|q(s)| d s\right) .
$$

Moreover, if the function $r \ln ^{2} r q(r)$ belongs to the class $L^{1}\left(0, r_{0}\right)$, then equation (4.1) has a solution $y^{(\operatorname{sing})}(r)$ satisfying the relation

$$
y^{(\operatorname{sing})}(r)=\ln r+o(1), \quad r \rightarrow 0 .
$$

In this case any bounded solution of equation (4.1) coincides (up to a constant factor) with the regular solution $y^{(r e g)}(r)$.

Proof. We construct the function $y^{(r e g)}(r)$ as the solution of the Volterra integral equation

$$
y^{(r e g)}(r)=y_{0}^{(r e g)}(r)+\varkappa_{m} \int_{0}^{r} s\left(y_{0}^{(r e g)}(r) y_{0}^{(s i n g)}(s)-y_{0}^{(r e g)}(s) y_{0}^{(s i n g)}(r)\right) q(s) y^{(r e g)}(s) d s
$$


where $\varkappa_{m}=(2 m)^{-1}$ for $m \neq 0$ and $\varkappa_{0}=-1$. Differentiating it twicely, we see that $y^{(r e g)}(r)$ satisfies equation (4.1). Equation (4.6) can be solved by iterations, that is

$$
y^{(r e g)}(r)=\sum_{n=0}^{\infty} y_{n}^{(r e g)}(r) .
$$

Hereby the $n^{\text {th }}$-iteration obeys the bound

$$
\left|y_{n}^{(r e g)}(r)\right| \leq \frac{C^{n}}{n !} r^{m}\left(\int_{0}^{r} s|q(s)| d s\right)^{n}
$$

if $m \neq 0$; if $m=0$, then $s|q(s)|$ should be replaced by $s|\ln s||q(s)|$. This ensures the convergence of series (4.7) as well as relation (4.2). Differentiating equation (4.6) and using (4.2), we get bounds (4.3) and (4.4) on the derivative of $y^{(r e g)}(r)$.

If $m=0$, we can construct the function $y^{(\operatorname{sing})}(r)$ as the solution of equation (4.6) where the first term, $y_{0}^{(r e g)}(r)$, in the right-hand side is replaced by $y_{0}^{(s i n g)}(r)$, that is

$$
y^{(s i n g)}(r)=\ln r+\int_{0}^{r} s \ln (r / s) q(s) y^{(s i n g)}(s) d s .
$$

This equation can again be solved by iterations which, in particular, implies estimate (4.5).

This result can be supplemented by the following

Lemma 4.2. Let $m \neq 0$, and let the function $r q^{2}(r)$ belong to the class $L^{1}\left(0, r_{0}\right)$. Assume additionally that $q=\bar{q}$. If $\psi$ is a solution of equation (4.1) from the class $L^{2}\left(\left(0, r_{0}\right) ; r d r\right)$, then it coincides (up to a constant factor) with the regular solution $y^{(r e g)}(r)$ and hence satisfies estimates (4.2) and (4.3).

Proof. Let us extend the function $q(r)$ to $\left(r_{0}, \infty\right)$ by zero, and let us consider the differential operator

$$
h y=-r^{-1}\left(r y^{\prime}\right)^{\prime}+m^{2} r^{-2} y+q(r) y
$$

in the space $L^{2}\left(\mathbb{R}_{+} ; r d r\right)$ on domain $C_{0}^{\infty}\left(\mathbb{R}_{+}\right)$. If $q=0$, we denote this operator by $h_{0}$. The operator $h_{0}$ is essentially self-adjoint. To prove the same for $h$, it suffices to check that

$$
\int_{\mathbb{R}_{+}} q^{2}(r)|f(r)|^{2} r d r \leq \varepsilon\left\|h_{0} f\right\|^{2}+C\|f\|^{2}, \quad f \in C_{0}^{\infty}\left(\mathbb{R}_{+}\right), \quad \varepsilon<1 .
$$

Let us use the estimate

$$
\begin{aligned}
& \int_{|x| \leq r_{0}} q^{2}(|x|)|u(x)|^{2} d x \leq \int_{|x| \leq r_{0}} q^{2}(|x|) d x \max _{x \in \mathbb{R}^{2}}|u(x)|^{2} \\
& \leq \varepsilon \int_{\mathbb{R}^{2}}|(\Delta u)(x)|^{2} d x+C \varepsilon^{-1} \int_{\mathbb{R}^{2}}|u(x)|^{2} d x, \quad \forall \varepsilon>0 .
\end{aligned}
$$

Restricting it on the subspace of functions $u(x)=f(r) e^{i m \theta}$, we obtain estimate (4.8) which implies that $h$ is essentially self-adjoint as well as $h_{0}$. Thus, equation (4.1) has at most one solution from $L^{2}\left(\left(0, r_{0}\right) ; r d r\right)$ which is necessarily proportional to $y^{(r e g)}(r)$. 
Now we are in a position to obtain a formula for the derivative $\lambda_{n, m}^{\prime}(p)$. In addition to our usual assumptions that $b(r)$ is not too singular at $r=0$, an integration-byparts marchinery requires that $b(r)$ does not vanish too rapidly as $r \rightarrow 0$. The precise conditions are formulated rather differently in the cases $m \neq 0$ and $m=0$. We start with the first case.

Theorem 4.3. Let $m \neq 0$. Suppose that $b \in C^{3}\left(\mathbb{R}_{+}\right)$and $b(r)>0, r \in \mathbb{R}_{+}$. Assume (2.10) and that $b(r)=O\left(e^{c r}\right)$ for some $c>0$ as $r \rightarrow \infty$. At $r=0$ we suppose that $b(r)=O\left(r^{-\gamma}\right)$ where $\gamma<3 / 2$. Moreover, we assume that for some $\beta<2|m|-1$

$$
\left|\left(b(r)^{-1}\right)^{(k)}\right| \leq C r^{-\beta-k}, \quad k=0,1,2,3, \quad r \rightarrow 0 .
$$

Put

$$
v(r)=r\left(r^{-1}\left(r b(r)^{-1}\right)^{\prime}\right)^{\prime}
$$

Then

$$
\begin{array}{r}
\lambda_{n, m}^{\prime}(p)=-2 \int_{0}^{\infty} r b^{-2}(r) b^{\prime}(r) \psi_{n, m}^{\prime}(r ; p)^{2} d r \\
-2^{-1} \int_{0}^{\infty} v^{\prime}(r) \psi_{n, m}^{2}(r ; p) d r+2 m^{2} \int_{0}^{\infty} r^{-2} b^{-1}(r) \psi_{n, m}^{2}(r ; p) d r
\end{array}
$$

where the eigenfunctions $\psi_{n, m}(r ; p)$ of the operator $H_{m}(p)$ are real and normalized, that is $\left\|\psi_{n, m}\right\|=1$.

Proof. In view of the equation

$$
(a(r)+p)^{2} \psi_{n, m}=r^{-1}\left(r \psi_{n, m}^{\prime}\right)^{\prime}-m^{2} r^{-2} \psi_{n, m}+\lambda_{n, m} \psi_{n, m}
$$

we can apply to the function $\psi_{n, m}$ the results of Lemmas 4.1 and 4.2 where $q(r)=$ $(a(r)+p)^{2}-\lambda_{n, m}$. Thus, Lemma 4.2 implies that $\psi_{n, m}(r ; p)=O\left(r^{|m|}\right)$ and $\psi_{n, m}^{\prime}(r ; p)=$ $O\left(r^{|m|-1}\right)$ as $r \rightarrow 0$ which ensures that non-integral terms disappear at $r=0$.

To prove the same for non-integral terms corresponding to $r \rightarrow \infty$, we use superexponential decay of eigenfunctions $\psi_{n, m}(r ; p)$ of the operators $H_{m}(p)$. This result is valid [14] (see also [6]) for all one-dimensional Schrödinger operators with discrete spectra. In view of the condition $a(r)=O\left(e^{c r}\right)$, it follows from equation (4.11) that the derivatives $\psi_{n, m}^{\prime}(r ; p)$ also decay super-exponentially.

Let us proceed from the formula of the first order perturbation theory (known as the Feynman-Hellman formula)

$$
\lambda_{n, m}^{\prime}(p)=\int_{0}^{\infty} \frac{\partial(a(r)+p)^{2}}{\partial p} \psi_{n, m}^{2}(r ; p) r d r=\int_{0}^{\infty} \frac{\partial(a(r)+p)^{2}}{\partial r} \psi_{n, m}^{2}(r ; p) \tau(r) d r
$$

where $\tau(r)=r b(r)^{-1}$. Using that $a(r)=O\left(r^{1-\gamma}\right)$ and $\tau(r)=O\left(r^{1-\beta}\right)$, we integrate by parts and get

$$
\lambda_{n, m}^{\prime}(p)=-\int_{0}^{\infty}(a(r)+p)^{2} \psi_{n, m}(r ; p)\left(\tau^{\prime}(r) \psi_{n, m}(r ; p)+2 \tau(r) \psi_{n, m}^{\prime}(r ; p)\right) d r .
$$


Now it follows from equation (4.11) that

$$
\begin{array}{r}
\lambda_{n, m}^{\prime}(p)=-\lambda_{n, m}(p) \int_{0}^{\infty}\left(\tau(r) \psi_{n, m}^{2}(r ; p)\right)^{\prime} d r+m^{2} \int_{0}^{\infty} r^{-2}\left(\tau(r) \psi_{n, m}^{2}(r ; p)\right)^{\prime} d r \\
-\int_{0}^{\infty} r^{-1}\left(r \psi_{n, m}^{\prime}(r ; p)\right)^{\prime}\left(\tau^{\prime}(r) \psi_{n, m}(r ; p)+2 \tau(r) \psi_{n, m}^{\prime}(r ; p)\right) d r
\end{array}
$$

By the condition $\tau(r) \psi_{n, m}^{2}(r ; p) \rightarrow 0$ as $r \rightarrow 0$, the first term in the right-hand side equals zero. In the second term we integrate by parts which yields

$$
\int_{0}^{\infty} r^{-2}\left(\tau(r) \psi_{n, m}^{2}(r ; p)\right)^{\prime} d r=2 \int_{0}^{\infty} r^{-3} \tau(r) \psi_{n, m}^{2}(r ; p) d r
$$

because $r^{-2} \tau(r) \psi_{n, m}^{2}(r ; p) \rightarrow 0$.

In the last integral in the right-hand side of (4.13), we also integrate by parts using that $\psi_{n, m}^{\prime}(r ; p) \psi_{n, m}(r ; p) \tau^{\prime}(r) \rightarrow 0$ as $r \rightarrow 0$. Thus, we have that

$$
\begin{aligned}
-\int_{0}^{\infty} r^{-1}\left(r \psi_{n, m}^{\prime}(r ; p)\right)^{\prime} \tau^{\prime}(r) \psi_{n, m}(r ; p) d r & =\int_{0}^{\infty} \tau^{\prime}(r) \psi_{n, m}^{\prime}(r ; p)^{2} d r \\
& +\int_{0}^{\infty} v(r) \psi_{n, m}^{\prime}(r ; p) \psi_{n, m}(r ; p) d r .
\end{aligned}
$$

The last integral in the right-hand side equals

$$
-2^{-1} \int_{0}^{\infty} v^{\prime}(r) \psi_{n, m}^{2}(r ; p) d r
$$

because $v(r) \psi_{n, m}^{2}(r ; p) \rightarrow 0$ as $r \rightarrow 0$. Similarly, we get that

$$
\begin{aligned}
-2 \int_{0}^{\infty} r^{-1}\left(r \psi_{n, m}^{\prime}(r ; p)\right)^{\prime} \tau(r) \psi_{n, m}^{\prime}(r ; p) d r & =-\int_{0}^{\infty} r^{-2} \tau(r) d\left(r \psi_{n, m}^{\prime}(r ; p)^{2}\right) \\
& =\int_{0}^{\infty} r^{2}\left(r^{-2} \tau(r)\right)^{\prime} \psi_{n, m}^{\prime}(r ; p)^{2} d r
\end{aligned}
$$

since $\tau(r) \psi_{n, m}^{\prime}(r ; p)^{2} \rightarrow 0$ as $r \rightarrow 0$. Putting the results obtained together, we arrive at representation (4.10).

Corollary 4.4. If $b^{\prime}(r) \leq 0$ and $r^{2} b(r) v^{\prime}(r) \leq 4 m^{2}$ for all $r \geq 0$, then $\lambda_{n, m}^{\prime}(p) \geq 0$ for all $p \in \mathbb{R}$ and $n$. If, moreover, one of these inequalities is strict on some interval, then $\lambda_{n, m}^{\prime}(p)>0$.

Corollary 4.5. If $b(r)=b_{0} r^{-\delta}, \delta \in[0,1]$, then $\tau(r)=b_{0}^{-1} r^{1+\delta}, v(r)=b_{0}^{-1}\left(\delta^{2}-1\right) r^{\delta-1}$ and

$$
\begin{array}{r}
\lambda_{n, m}^{\prime}(p)=2 b_{0}^{-1} \delta \int_{0}^{\infty} r^{\delta} \psi_{n, m}^{\prime}(r ; p)^{2} d r \\
+b_{0}^{-1}\left(2 m^{2}-2^{-1}(1-\delta)^{2}(1+\delta)\right) \int_{0}^{\infty} r^{-2+\delta} \psi_{n, m}^{2}(r ; p) d r
\end{array}
$$


For $b_{0}>0$, this expression is strictly positive (so that the functions $\lambda_{n, m}(p)$ are strictly increasing for all $p \in \mathbb{R})$ for $m \neq 0$ since $(1-\delta)^{2}(1+\delta) \leq 1$. Moreover, for $\delta=1$ this result is true for all $m \in \mathbb{Z}$.

In the case $m=0$ we consider for simplicity only fields (1.11).

Proposition 4.6. If $b(r)=b_{0} r^{-\delta}, \delta \in[0,1]$, then

$$
\begin{array}{r}
\lambda_{n, 0}^{\prime}(p)=2 b_{0}^{-1} \delta \int_{0}^{\infty} r^{\delta} \psi_{n, 0}^{\prime}(r ; p)^{2} d r \\
-b_{0}^{-1} 2^{-1}(1-\delta)^{2}(1+\delta) \int_{0}^{\infty} r^{-2+\delta}\left(\psi_{n, 0}^{2}(r ; p)-\psi_{n, 0}^{2}(0 ; p)\right) d r .
\end{array}
$$

If $b_{0}>0$ and $\delta=1$, then $\lambda_{n, 0}^{\prime}(p)>0$ for all $p \in \mathbb{R}$.

Proof. Let us proceed again from formula (4.12). We use now that the function $\psi_{n, 0}(|x| ; p)$ of $x \in \mathbb{R}^{2}$ belongs to the Sobolev class $\mathrm{H}_{l o c}^{2}\left(\mathbb{R}^{2}\right)$, and therefore $\psi_{n, 0}(r ; p)$ has a finite limit as $r \rightarrow 0$. Thus, by Lemma $4.1 \psi_{n, 0}^{\prime}(r ; p)=O\left(r^{1-\varepsilon}\right)$ for any $\varepsilon>0$ as $r \rightarrow 0$. These results allow us to intergrate by parts as in the case $m \neq 0$. The only difference is with the second integral in the right-hand side of (4.14). Now $v(r)=b_{0}^{-1}\left(\delta^{2}-1\right) r^{\delta-1}$ and this integral equals

$$
\begin{aligned}
\int_{0}^{\infty} v(r) \psi_{n, 0}^{\prime}(r ; p) \psi_{n, 0}(r ; p) d r & =2^{-1} \int_{0}^{\infty} v(r) d\left(\psi_{n, 0}^{2}(r ; p)-\psi_{n, 0}^{2}(0 ; p)\right) \\
& =-2^{-1} \int_{0}^{\infty} v^{\prime}(r)\left(\psi_{n, 0}^{2}(r ; p)-\psi_{n, 0}^{2}(0 ; p)\right) d r
\end{aligned}
$$

because $v(r)\left(\psi_{n, 0}^{2}(r ; p)-\psi_{n, 0}^{2}(0 ; p)\right)$ as $r \rightarrow 0$.

4.2. In this subsection we show that for linear potentials, that is for magnetic fields not depending on $r$, all eigenvalues $\lambda_{n, 0}(p), n \in \mathbb{N}$, of the operator $H_{0}(p)$ are not monotonous functions of $p \in \mathbb{R}$. We follow closely the proof of the first part of Proposition 3.6. However we now use that eigenfunctions of the harmonic oscillator decay faster than any power of $r^{-1}$ at infinity (actually, they decay super-exponentially).

Proposition 4.7. Assume that for sufficiently large $r$

$$
b(r)=b_{0}>0 .
$$

Then, for all $n \in \mathbb{N}$, some $\gamma_{n}>0$ and sufficiently large $k>0$, we have

$$
\lambda_{n, 0}(-k) \leq(2 n-1) b_{0}-\gamma_{n} k^{-2} .
$$

Proof. Let $\zeta$ be the same function as in the proof of the first part of Proposition 3.6. We set $\rho_{k}=b_{0}^{-1} k, \gamma_{k}=2 b_{0}^{1 / 2} k^{-1}$ and define the functions $\zeta(r ; k)$ and $\varphi_{j}(r ; k)$ by formulas (3.31) and (3.32), respectively. It suffices to check that

$$
\left\langle L_{0}(-k) \varphi(k), \varphi(k)\right\rangle \leq 2 n-1-\gamma_{n} k^{-2} .
$$

for sufficiently large $k$ and all normalized functions from subspace (3.16). Let us proceed from formula (3.35). Since the functions $\psi_{j}(x ; k)$ decay faster than any power of $|x|^{-1}$ 
as $|x| \rightarrow \infty$, the term $o(1)$ in (3.33) is actually $O\left(k^{-\infty}\right)$. Similarly, estimate (3.36) can be formulated in a more precise form as

$$
\left\|\psi(k) \zeta^{\prime}(k)\right\|^{2}=O\left(k^{-\infty}\right) .
$$

Since $r \leq 2^{-1} 3 k$ on the support of $\varphi(k)$, we have that

$$
\left\|r^{-1} \psi(k)\right\|^{2} \geq(2 / 3)^{2} k^{-2} .
$$

Now function (3.34) is zero if $r$ and $k$ are large enough. Therefore equation (3.38) yields the exact equality

$$
\operatorname{Re}\left\langle-\psi^{\prime \prime}(k)+\left(b_{0} r-k\right)^{2} \psi(k), \psi(k) \zeta^{2}(k)\right\rangle=b_{0} \sum_{j, l=1}^{n}(2 j-1) c_{j} \bar{c}_{l}\left\langle\varphi_{j}(k), \varphi_{l}(k)\right\rangle
$$

(cf. (3.41) ). Up to terms $O\left(k^{-\infty}\right)$, the right-hand side here is estimated by $b_{0}(2 n-1)$. Together with (4.18) and (4.19), this implies estimate (4.17).

Combining relations (3.25) and (4.16), we see that the eigenvalues $\lambda_{n, 0}(p)$ tend as $p \rightarrow-\infty$ to their limits $(2 n-1) b_{0}$ from below. On the other hand, according to (3.5) $\lambda_{n, 0}(p) \rightarrow \infty$ as $p \rightarrow \infty$. Thus, all functions $\lambda_{n, 0}(p)$ have necessarily local minima. We can obtain an additional information using the following elementary

Lemma 4.8. Suppose that (4.15) is satisfied for all $r>0$ and that $a(r)=b_{0} r$. Then

$$
\lambda_{n, m}(0)=2 b_{0}(2 n-1+|m|)
$$

for all $n \in \mathbb{N}$ and $m \in \mathbb{Z}$.

Proof. Let us consider the two-dimensional harmonic oscillator $\mathbf{T}=-\Delta+b_{0}^{2}\left(x_{1}^{2}+x_{2}^{2}\right)$. Separating the variables $x_{1}, x_{2}$, we see that its spectrum consists of the eigenvalues $2 b_{0}\left(l_{1}+l_{2}-1\right)$ where $l_{1}, l_{2} \in \mathbb{N}$. It follows that the operator $\mathbf{T}$ has the eigenvalues $2 b_{0} j, j \in \mathbb{N}$, of multiplicity $j$. On the other hand, separating the variables in the polar coordinates, we see that the spectrum of $\mathbf{T}$ consists of the eigenvalues $\lambda_{n, m}(0)$ of the operators $H_{m}(0)$. For the proof of (4.20) we take into account that all eigenvalues $\lambda_{n, m}(0)$ are simple and that $\lambda_{n, m+1}(0)>\lambda_{n, m}(0)$ for all $n$ and $m \geq 0$. Clearly, the operator $H_{0}(0)$ has an eigenvalue $2 b_{0} j$ if and only if its multiplicity $j$ is odd. This gives formula (4.20) for $m=0$. We shall show that for every $j \in \mathbb{N}$

$$
\lambda_{1, j-1}(0)=\lambda_{2, j-3}(0)=\ldots=\lambda_{2,-j+3}(0)=\lambda_{1,-j+1}(0)=2 b_{0} j
$$

which is equivalent to formula (4.20) for all $\mathrm{m}$. Let us choose some $j_{0}$ and suppose that (4.21) holds for all $j \leq j_{0}$. Then we check it for $j=j_{0}+1$. First we remark that if an operator $H_{m}(0)$ for some $m>0$ has $n$ eigenvalues in the interval $\left[2 b_{0}, 2 b_{0}\left(j_{0}+1\right)\right]$, then the operator $H_{m-1}(0)$ has at least $n$ eigenvalues in the interval $\left[2 b_{0}, 2 b_{0} j_{0}\right]$. Then using (4.21) for $j \leq j_{0}$, we see that if an operator $H_{m}(0)$ has the eigenvalue $2 b_{0}\left(j_{0}+1\right)$, then necessarily the operator $H_{m-1}(0)$ has the eigenvalue $2 b_{0} j_{0}$. Therefore according to (4.21) for $j=j_{0}$, only the operators $H_{m}(0)$ with $m=j_{0}, j_{0}-2, \ldots,-j_{0}+2,-j_{0}$ might have the eigenvalue $2 b_{0}\left(j_{0}+1\right)$. There are $j_{0}+1$ of such operators and the multiplicity of this eigenvalue equals $j_{0}+1$. Thus, all the operators $H_{m}(0)$ for $m=j_{0}, j_{0}-2, \ldots,-j_{0}+2,-j_{0}$ and only for such $m$ have the eigenvalue $2 b_{0}\left(j_{0}+1\right)$. This proves (4.21) for $j_{0}+1$. 
Comparing this result with (3.27), we see that, for potentials $a(r)=b_{0} r$,

$$
\lim _{p \rightarrow-\infty} \lambda_{n, m}(p)=b_{0}(2 n-1)<2 b_{0}(2 n-1+|m|)=\lambda_{n, m}(0) .
$$

Together with (4.16), this implies that the functions $\lambda_{n, 0}(p)$ have negative local minima. Thus, we get the following

Theorem 4.9. Under the hypotheses of Proposition 4.7 the eigenvalues $\lambda_{n, 0}(p), n \in \mathbb{N}$, of the operator $H_{0}(p)$ are not monotonous functions of $p \in \mathbb{R}$. Moreover, if (4.15) is satisfied for all $r>0$ and $a(r)=b_{0} r$, then the functions $\lambda_{n, 0}(p)$ lose their monotonicity for $p<0$.

We do not know how many minima have the functions $\lambda_{n, 0}(p)$.

The problem of monotonicity of the eigenvalues $\lambda_{n, 0}(p)$ for fields $b(r)=b_{0} r^{-\delta}$ where $\delta \in(0,1)$ remains also open.

4.3. In a somewhat similar situation the break down of monotonicity of group velocities was exhibited in [7]. In this paper one considers the Schrödinger operator $\mathbf{H}^{(N)}=-\frac{\partial^{2}}{\partial x^{2}}+\left(i \frac{\partial}{\partial y}-b x\right)^{2}$ with constant magnetic field $b>0$, defined on the semi-plane $\left\{(x, y) \in \mathbb{R}^{2}: x>0\right\}$ with the Neumann boundary condition at $x=0$. Let $H^{(N)}(p)=-d^{2} / d x^{2}+(b x+p)^{2}, p \in \mathbb{R}$, be the self-adjoint operator in the space $L^{2}\left(\mathbb{R}_{+}\right)$ corresponding to the boundary condition $u^{\prime}(0)=0$. Then the operator $\mathbf{H}^{(N)}$ is unitarily equivalent under the partial Fourier transform with respect to $y$, to the direct integral $\int_{\mathbb{R}}^{\oplus} H^{(N)}(p) d p$. It is shown in [7, Section 4.3] that the lowest eigenvalue $\mu_{1}(p)$ of $H^{(N)}(p)$ is not monotonous for $p<0$. This follows from the inequality $\mu_{1}^{\prime}(0)>0$ proven! in $[3]$ and the relations

$$
\lim _{p \rightarrow-\infty} \mu_{1}(p)=\mu_{1}(0)=b
$$

Our proof of non-monotonicity of the functions $\lambda_{n, 0}(p)$ is essentially different since in contrast with (4.22) we have $\lim _{p \rightarrow-\infty} \lambda_{n, 0}(p)<\lambda_{n, 0}(0)$.

\section{Asymptotic time evolution}

5.1. Combined with the stationary phase method, the spectral analysis of the operators $\mathbf{H}=\mathbf{H}(a)$ allows us to find the asymptotics for large $t$ of solutions $u(t)=$ $\exp (-i \mathbf{H} t) u_{0}$ of the time dependent Schrödinger equation. It follows from (1.2) that

$$
\overline{\exp (-i \mathbf{H}(a) t) u_{0}}=\exp (i \mathbf{H}(-a) t) \overline{u_{0}} .
$$

Therefore it suffices to consider the case $a(r) \rightarrow+\infty$. Moreover, on every subspace $\mathfrak{H}_{m}$ with a fixed magnetic quantum number $m$, the problem reduces to the asymptotics of the function $u(t)=\exp \left(-i \mathbf{H}_{m} t\right) u_{0}$.

Let us proceed from decomposition (2.12). Suppose that $\mathcal{F}_{m} u_{0} \in \operatorname{Ran} \Psi_{n, m}$. Then $(\operatorname{see}(2.11))$

$$
\left(\mathcal{F}_{m} u_{0}\right)(r, p)=\psi_{n, m}(r, p) f(p)
$$

\footnotetext{
${ }^{1}$ Note that in [3] and [7] the parameter $p$ is chosen with the opposite sign.
} 
where $f=\Psi_{n, m}^{*} \mathcal{F}_{m} u_{0}$ and $u(t)=\mathcal{F}_{m}^{*} \Psi_{n, m} e^{-i \Lambda_{n, m} t} f$, that is

$$
u_{n, m}\left(r, x_{3}, t\right)=(2 \pi)^{-1 / 2} \int_{-\infty}^{\infty} e^{i p x_{3}-i \lambda_{n, m}(p) t} \psi_{n, m}(r, p) f(p) d p .
$$

The analytic function $\lambda_{n, m}^{\prime \prime}(p)$ might have only a countable set of zeros $p_{n, m, l}$ with possible accumulations at $\pm \infty$ only. The function $\lambda_{n, m}^{\prime}(p)$ is monotone on every interval $\left(p_{n, m, l}, p_{n, m, l+1}\right)$ and takes there all values between $\lambda_{n, m}^{\prime}\left(p_{n, m, l}\right)=: \alpha_{n, m, l}$ and $\lambda_{n, m}^{\prime}\left(p_{n, m, l+1}\right)=: \beta_{n, m, l}$. We consider the asymptotics of integral (5.2) on each of the subspaces $L^{2}\left(p_{n, m, l}, p_{n, m, l+1}\right)$ separately. Let us set $\gamma=x_{3} t^{-1}$. First we suppose that $f \in C_{0}^{\infty}\left(p_{n, m, l}, p_{n, m, l+1}\right)$. The stationary points of integral (5.2) are determined by the equation

$$
\lambda_{n, m}^{\prime}(p)=\gamma
$$

If $\gamma \notin\left(\alpha_{n, m, l}, \beta_{n, m, l}\right)$, it does not have solutions from the interval $\left(p_{n, m, l}, p_{n, m, l+1}\right)$. Therefore integrating directly by parts, we find that function (5.2) decays in this region of $x_{3} / t$ faster than any power of $\left(\left|x_{3}\right|+|t|\right)^{-1}$ (and $r$ ). If $\gamma \in\left(\alpha_{n, m, l}, \beta_{n, m, l}\right)$, then on the interval $\left(p_{n, m, l}, p_{n, m, l+1}\right)$ equation (5.3) has a unique solution which we denote by $\nu_{n, m, l}(\gamma)$. Let us set

$$
\Phi_{n, m, l}(\gamma)=\nu_{n, m, l}(\gamma) \gamma-\lambda_{n, m}\left(\nu_{n, m, l}(\gamma)\right)
$$

and denote by $\chi_{n, m, l}$ the characteristic function of the interval $\left(\alpha_{n, m, l}, \beta_{n, m, l}\right)$. For $\gamma$ from this interval, we apply the stationary phase method to integral (5.2) which yields

$$
\begin{gathered}
u\left(r, x_{3}, t\right)=\tau_{n, m, l}^{( \pm)} e^{i \Phi_{n, m, l}(\gamma) t} \psi_{n, m}\left(r, \nu_{n, m, l}(\gamma)\right)\left|\lambda_{n, m}^{\prime \prime}\left(\nu_{n, m, l}(\gamma)\right)\right|^{-1 / 2} \\
\times f\left(\nu_{n, m, l}(\gamma)\right) \chi_{n, m, l}(\gamma)|t|^{-1 / 2}+u_{\infty}\left(r, x_{3}, t\right), \quad \gamma=x_{3} t^{-1}, \quad t \rightarrow \pm \infty,
\end{gathered}
$$

where $\tau_{n, m, l}^{( \pm)}=e^{\mp \pi i \operatorname{sgn}\left(\lambda_{n, m}^{\prime \prime}(p)\right) / 4}$ for $p \in\left(p_{n, m, l}, p_{n, m, l+1}\right)$ and

$$
\lim _{t \rightarrow \pm \infty}\left\|u_{\infty}(\cdot, t)\right\|=0 \text {. }
$$

Since the norm in the space $\mathfrak{H}$ of the first term in the right-hand side of (5.4) equals the norm of $f$ in the space $L^{2}\left(p_{n, m, l}, p_{n, m, l+1}\right)$, asymptotics (5.4) extends to all functions (5.1) with an arbitary $f \in L^{2}\left(p_{n, m, l}, p_{n, m, l+1}\right)$. Thus, we have proven

Theorem 5.1. Assume (2.4) and (2.10). Let $u(t)=\exp \left(-i \mathbf{H}_{m} t\right) u_{0}$ where $u_{0}$ satisfies (5.1) with $f \in L^{2}\left(p_{n, m, l}, p_{n, m, l+1}\right)$. Then the asymptotics as $t \rightarrow \pm \infty$ of this function is given by relations (5.4), (5.5).

Of course asymptotics (5.4), (5.5) extends automatically to all $f \in L^{2}(\mathbb{R})$ with compact support and to linear of functions $\psi_{n, m}(r, p) f_{n}(p)$ over different $n$.

By virtue of formulas (5.4), (5.5) a quantum particle in magnetic field (1.5) remains localized in the $\left(x_{1}, x_{2}\right)$-plane but propagates in the $x_{3}$-direction. If $f \in L^{2}\left(p_{n, m, l}, p_{n, m, l+1}\right)$, then a particle "lives" as $|t| \rightarrow \infty$ in the region where $x_{3} \in\left(\alpha_{n, m, l} t, \beta_{n, m, l} t\right)$. In particular, if $\lambda^{\prime}(p)>0\left(\lambda^{\prime}(p)<0\right)$ for $p \in\left(p_{n, m, l}, p_{n, m, l+1}\right)$, then a particle propagates in the positive (negative) direction as $t \rightarrow+\infty$. Thus, according to Corollary 4.5 if $b(r)=b_{0} r^{-\delta}, \delta \in[0,1], b_{0}>0$, then a particle with the magnetic quantum number $m \neq 0$ propagates always in the positive direction of the $x_{3}$-axis. If $\delta=1$, then this 
result remains true from all $m$. On the contrary, if $\delta=0$ and $m=0$, then a particle will propagate in a negative direction for some interval of momenta $p$.

5.2. Theorem 5.1 implies the existence of asymptotic velocity in the $x_{3}$-direction. The corresponding operator is defined by the equation (cf. (2.12)

$$
\mathbf{H}_{m}^{\prime}=\bigoplus_{n \in \mathbb{N}} \mathcal{F}_{m}^{*} \Psi_{n, m} \Lambda_{n, m}^{\prime} \Psi_{n, m}^{*} \mathcal{F}_{m}
$$

where $\Lambda_{n, m}^{\prime}$ are the operators of multiplication by the functions $\lambda_{n, m}^{\prime}(p)$. To put it differently, the operator $\mathbf{H}_{m}^{\prime}$ acts as multiplication by $\lambda_{n, m}^{\prime}(p)$ in the spectral representation of the operator $\mathbf{H}_{m}$ where it acts as multiplication by the functions $\lambda_{n, m}(p)$.

Proposition 5.2. Assume (2.4) and (2.10). Then, for an arbitrary bounded function $\mathcal{Q}$

$$
\mathrm{s}^{-} \lim _{|t| \rightarrow \infty} \exp \left(i \mathbf{H}_{m} t\right) \mathcal{Q}\left(x_{3} / t\right) \exp \left(-i \mathbf{H}_{m} t\right)=\mathcal{Q}\left(\mathbf{H}_{m}^{\prime}\right)
$$

(in particular, the strong limit in the left-hand side exists).

Proof. We shall check that for all $u_{0} \in \mathcal{H}_{m}$

$$
\lim _{|t| \rightarrow \infty}\left\|\mathcal{Q}\left(x_{3} / t\right) \exp \left(-i \mathbf{H}_{m} t\right) u_{0}-\exp \left(-i \mathbf{H}_{m} t\right) \mathcal{Q}\left(\mathbf{H}_{m}^{\prime}\right) u_{0}\right\|=0
$$

which is equivalent to relation (5.6). Remark that if $u_{0}$ satisfies (5.1), then

$$
\left(\Psi_{n, m} \mathcal{Q}\left(\mathbf{H}_{m}^{\prime}\right) u_{0}\right)(r, p)=\psi_{n, m}(r, p) \mathcal{Q}\left(\lambda_{n, m}^{\prime}(p)\right) f(p) .
$$

It suffices to prove (5.7) on a dense set of elements $u_{0}$ such that equality (5.1) is true with $f \in L^{2}\left(p_{n, m, l}, p_{n, m, l+1}\right)$. Applying the operator $\mathcal{Q}\left(x_{3} / t\right)$ to asymptotic relation (5.4), we see that the asymptotics of $\mathcal{Q}\left(x_{3} / t\right) \exp \left(-i \mathbf{H}_{m} t\right) u_{0}$ is given again by formula (5.4) where the function $f\left(\nu_{n, m, l}(\gamma)\right)$ in the right-hand side is replaced by the function $\mathcal{Q}(\gamma) f\left(\nu_{n, m, l}(\gamma)\right)$. Similarly, it follows from Theorem 5.1 and relation (5.8) that the asymptotics of $\exp \left(-i \mathbf{H}_{m} t\right) \mathcal{Q}\left(\mathbf{H}_{m}^{\prime}\right) u_{0}$ is given by formula (15.4) where the function $f\left(\nu_{n, m, l}(\gamma)\right)$ in the right-hand side is replaced by the function $\mathcal{Q}\left(\lambda_{n, m}^{\prime}\left(\nu_{n, m, l}(\gamma)\right)\right) f\left(\nu_{n, m, l}(\gamma)\right)$. So for the proof of (5.7), it remains to take equation (5.3) into account.

Relation (5.6) shows that $\mathbf{H}_{m}^{\prime}$ can naturally be interpreted as the operator of asymptotic velocity in the $x_{3}$-direction.

Similar results concerning the Iwatsuka model (see [8] or [2]) have been obtained in [11].

Numerous useful discussions with Georgi Raikov as well as a financial support by the Chilean Science Foundation Fondecyt under Grant 7050263 are gratefully acknowledged.

\section{REFERENCES}

[1] Y. Aharonov, D. Bohm, Significance of electromagnetic potential in the quantum theory, Phys. Rev. 115 (1959), 485-491.

[2] H. Cycon, R. Froese, W. Kirsch, B. Simon, Schrödinger Operators with Application to Quantum Mechanics and Global Geometry, Texts and Monographs in Physics, Springer-Verlag, Berlin, Heidelberg, New York, 1987.

[3] M. Dauge, B. Helffer, Eigenvalues variation. I. Neumann problem for Sturm-Liouville operators, J. Diff. Eq. 104 (1993), 243-262. 
[4] S. De Bièvre, J. V. Pulé, Propagating edge states for a magnetic Hamiltonian, Math. Phys. Electron. J. 5 (1999), Paper 3, 17 pp.

[5] V. GeĬler, M. Senatorov, The structure of the spectrum of the Schrödinger operator with a magnetic field in a strip, and finite-gap potentials, Sb. Math. 188 (1997), 657-669.

[6] I. M. Glazman, Direct methods of qualitative spectral analysis of singular differential operators, Moscow, Fizmatgiz, 1963 (Russian).

[7] B. HeLfFer, Introduction to semi-classical methods for the Schrödinger operator with magnetic field. Vienna version, Lecture notes of a course given at the ESI, 2006, available at http://www.math.u-psud.fr/ helffer/syrievienne2006.pdf.

[8] A. Iwatsuka, Examples of absolutely continuous Schrödinger operators in magnetic fields, Publ. Res. Inst. Math. Sci. 21 (1985), no. 2, 385-401.

[9] T. Kato, Perturbation Theory for Linear Operators, Die Grundlehren der mathematischen Wissenschaften, 132 Springer-Verlag New York, Inc., New York 1966.

[10] L. D. Landau, E. M. Lifshitz, Quantum mechanics, Pergamon Press, 1965.

[11] M. Măntoiu, R. Purice, Some propagation properties of the Iwatsuka model, Comm. Math. Phys. 188 (1997), 691-708.

[12] K. Miller, B. Simon, Quantum magnetic Hamiltonians with remarkable spectral properties, Phys. Rev. Lett. 44 (1980), 1706-1707.

[13] M. Reed, B. Simon, Methods of Modern Mathematical Physics, IV. Analysis of Operators, Academic Press, New York, 1978.

[14] I. E. Shnol', On the behavior of eigenfunctions of the Schrödinger equation, Matem. Sb. 42 (1957), 273-286 (in Russian).

[15] D. Yafaev, A particle in a magnetic field of an infinite rectilinear current, Math. Phys. Anal. Geom. 6 (2003), 219-230.

[16] D. Yafaev, Scattering by magnetic fields, St. Petersburg Math. J. 17 No. 5 (2006), 875-895.

Département de mathématiques, Université de Rennes I, Campus Beaulieu, 35042 Rennes, FRANCE, yafaev@univ-rennes1.fr

IRMAR, Université de Rennes I, Campus de Beaulieu, 35042 Rennes Cedex, FRANCE E-mail address: yafaev@univ-rennes1.fr 